herabsetzung erleiden (Pugliese und Luzzatti, Banti und Furno, Strisower und Goldschmidt, Widal und ABRAMI, EPPINGER). Es hat jedoch auch nicht an Untersuchern gefehlt, die ein entgegengesetztes Resultat erhielten, das aber immer auch zugunsten einer zerstörenden Tätigkeit der Milz verwendet wurde, indem man voraussetzte, $\mathrm{daB}$ nur die resistenteren Erythrocyten imstande waren, das Organ zu passieren (Hammarsten, Gabbi, Chalier und Chardet).

Vom Standpunkt der BRINkmannschen Untersuchungen aus ist die bisher gebrauchte Methodik nicht einwandfrei. Aus diesen Untersuchungen ergab sich nämlich, daß Blutkörperchen, welche vor der Resistenzbestimmung gewaschen werden mit isotonischer NaCl-Lösung geschädigt werden, indem das $\mathrm{NaCl}$ einen sog. lyotropen Effekt hat auf die Oberflächenkolloide der Erythrocyten, d. h. also da $\beta$ die reine Kochsalzlösung eine verflüssigende Wirkung hat auf die Oberfläche. Diese Wirkung ergibt sich $u$. a. daraus, daß nach wiederholtem Waschen mit isotonischer Kochsalzlösung die Erythrocyten selbst in diesem isotonischen Milieu hämolysieren. Es ist also erwünscht, als Waschflüssigkeit nur äquilibrierte Salzlösung $z u$ benützen, und zwar eine $\mathrm{Ca}$ haltige Lösung (modifizierte Ringerlösung). Aber auch wenn man zur Resistenzbestimmung nur ungewaschene Blutkörperchen verwendet, so ist es konsequent, auch dann den lyotropen Effekt auszuschaiten und diese Bestimmungen in hypotonischen äquilibrierten -Lösungen vorzunchmen.

Wir haben nun auf diese Weise die osmotische Resistenz der Blutkörperchen aus der Milzvene bestimmt. Um Milzblutkörperchen in genügender Weise zu erlangen, wurden diese der Milz des eben getöteten Schafes entnommen und

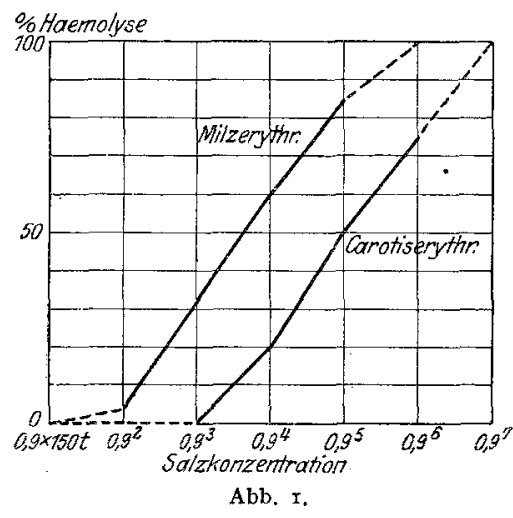
mit Carotisblutkörperchen verglichen. Von beiden Arten Blutkörperchen wurde jetzt auf der üblichen Weise, jedoch in für die Erythrocyten geeignete. Flüssigkeit, die osmotische Resistenz bestimmt. In einer großen Anzahl Versuche fanden wir ausnahmslos eine mehr oder weniger starke Resistenzerniedrigung der Milzblutkörperchen, verglichen mit den Carotisblutkörperchen. In der nebenstehenden Figur sind die Mittelwerte aus 20 Bestimmungen graphisch dargestellt; es ist darin also ein ganz willkürlicher Fall zu ersehen.

Wir können also den Autoren, die schon eine Resistenzherabsetzung in der Milz feststellen konnten, völlig beistimmen und betrachten diese Erscheinung als ein sicheres Zeichen einer hämolytischen Funktion der Milz, dies in bezug auf die Bedeutung, welche der osmotischen Resistenz der Erythrocyten zukommt ${ }^{1}$.

Wir haben nun diese Milzfunktion näher studiert, indem wir auch die Resistenz đer mit äquilibrierter Salzlösung gewaschenen Milzblutkörperchen mit der Resistenz der Carotisblutkörperchen verglichen. Aus den obenerwähnten Untersuchungen BRINKMANs ist nämlich weiter hervorgegangen, daß man die osmotische Resistenz der Blutkörperchen erhöht, wenn man sie einige Male mit isotonischer äquilibrierter Salzlösung wäscht (im Gegenteil wird durch đas Waschen der Erythrocyten mit isotonischer Kochsalzlösung die Resistenz erniedrigt - SNAPPER). Durch das Waschen mitäquilibrierter Salzlösung wird nämlich eine hämolytische Substanz (Phosphatid) aus der Oberfläche ausgespült, die immer in ihrer Wirkung gehemmt wird von dem Cholesterin der Körperchen.

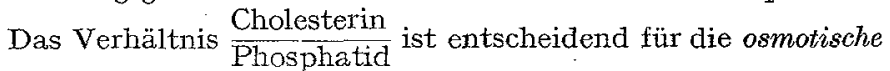

1) BRINKMAN, Résistance osmotique et phosphatides du Sang, Diss, Groningen $x 922$.
Resistenz der Erythrocyten. Sollte nun die hämolytische Funktion der Milz sich äußern in einer Erniedrigung dieses Verhältnisses $\frac{\text { Cholesterin }}{\text { Phosphatid }}$, so könnte man erwarten, daß nach dem Auswaschen dieses Komplexes die beiden Blutarten wieder ähnliche Resistenzwerte zeigten. Diese Voraussetzing hat sich nun völlig bestätigt, wie aus der Abb. $2 \mathrm{zu}$ ersehen ist.

Wir müssen uns also das Wesen der Milzfunktion folgendermaßen denken: alle Blutkörperchen, die in die Milz eintreten, erleiden darin eine Resistenzherabsetzung, d. h. sie kommen der Hämolyse näher. Die schwächeren gehen an der Wirkung zugrunde, die stärkeren kommen abgeschwächt wieder zur Milz heraus.

Ein völlig gleiches Resultat

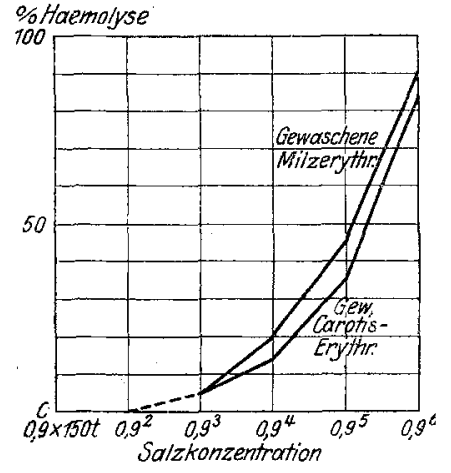

Abb. 2. erhielten wir mit einer Resistenzbestimmung der Erythrocyten aus der menschlichen Milzvene: auch hier war die Resistenz der Milzblutkörperchen niedriger als diejenige der Erythrocyten aus dem peripheren Blut, während nach dem Waschen fast jeder Unterschied verschwand. Es wäre wünschenswert, durch weitere Bestimmungen, wie sie bei evtl. Milzexstirpationen leicht vorzunehmen sind, diesen Befund näher zu bestätigen.

Kontrollbestimmungen an der Niere ergaben Resistenzunterschiede, die sich nicht erhöhen über die Unterschiede zwischen arteriellem und venösem Blut im allgemeinen. (Physiologisches Institut der Reichsuniversität Groningen.)

\section{ZUR FRAGE DER THEORIE UND PRAKTISCHEN BRAUCHBARKEIT VON WIDALS HÄMOKLASTISCHER KRISE ${ }^{1}$ ). \\ Von}

HANS EISENSTÄDT,

WIDAL hatte seine Leberfunktionsprüfung, die hämoklastische Krise, theoretisch damit begründet, da.B bei Leberkranken eine Störung der hypothetischen „, fonction protéo-

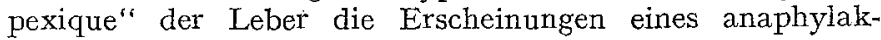
tischen Schocks im kleinen hervorrufe.

-Diese Theorie ist vielfach erschüttert worden. Die Spezifität der Prüfung erscheint fraglich; sowohl zeigen offensichtlich Leberkranke vereinzelt keine ,hämoklastische Krise" als auch tritt sie oft bei Nicht-Leberkranken (SÖMJÉN, BAUER, Dresel-Lewy u. a.) und allen Säuglingen und Kindern auf (Schiff u. Stransky). Auch die Behauptung Widals, daß es sich um eine Eiweißfunktionsprüfung der Leber handelt, erscheint zweifelhaft. Denn die Krise läßt sich auch durch andere Maßnahmen als durch Milchtrinken (Eiweiß) herbeiführen z. B. durch Aufnahme von Kohlenhydraten und Fetten (RetzlafF, Jungmann u. a.), durch Massage der Leber (SöMJÉN), durch Aufnahme von Wasser allein (MAUTNER u. CoRI). Mit Unrecht weist ferner der Name "Hämoklasie" auf eine Blutzerstörung hin, während man in dem Sinken der Leukocyten nur eine Verteilungsleukopenie sehen kann (Worms-Schreiber, F. Glaser). Glaser faßt die hämoklastische Krise überhaupt als "abdominellen Vagusreflex" auf, der bei erhöhtem Vagustonus zustande kommt. Erhöht ist der Vagustonus z. B. im jugendlichen Alter, bei HysteroNeurasthenie, bei Ikterus (!).

Trotz dieser Bedenken wurde mit geringen Ausnahmen (Worms-SchreIber, ERdMANN) bis in die letzte Zeit die klinische Brauchbarkeit der WIDALschen hämoklastischen Krise betont. An Leberkranken, anderen Kranken und Gesunden untersuchte ich nun ebenfalls daraufhin, und zwar lag 
mir daran, den Ablauf der Kurven für den (bisher wenig untersuchten) Bluteiweißgehalt und Blutdruck, die Leukocytenzahl und -verschiebung gleichzeitig festzustellen. Es ergab sich folgendes Resultat:

I. Die Leukocytenzahlen: Ein Steigen (= alimentäre Leukocytose) kommt auch bei Leberkranken vor; Sinken (= alimentäre Leukopenie $=$,Hämoklasie" ) nicht nur bei Leberkranken, sondern auch bei anderen Kranken und gesunden Erwachsenen (20-23 Jahre alt) ohne jede Leberschädigung. Findet sich ein Leukocytensturz bei Leberkranken ein, so so geht erstens Intensität der Lebererkrankung und Stärke der Leukocytensenkung nicht parallel, zweitens ist die Differenz der Leukocytenzahlen oft so gering (weniger als I7\% der Ausgangssumme), daß sie durchaus im Bereich der technischen Fehlerquellen und der Tagesschwankungen liegt. Für die Deutung des Resultates kommt nur die Glaser sche Theorie (siehe oben) in Betracht.

II. Das Blutbild: a) Bei den Neutrophilen und Lymphocyten keine "Umkehrung der Leukocytenformel“ (WIDAL), sondern sowohl bei alimentärer Leukopenie wie Leukocytose relatives Ansteigen der Neutrophilen und Sinken der Lymphocyten, d. h. in absoluten Zahlen ausgedrückt: bei gleichzeitigem Sinken der Leukocyten überhaupt (Verdauungsleukopenie) beteiligen sich am Leukocytensturz schneller und ausgiebiger die Lymphocyten; bei gleichzeitigem Steigen der Leukocyten (Verdauungsleakocytose) reagieren schneller und ausgiebiger die Neutrophilen. - b) Für das Verhalten der Monocyten und Eosinophilen läßt sich keine Regel aufstellen.

III. Der Blutdruck zeigte im allgemeinen außerordentlich geringe Schwankungen, oft nur um wenige Millimeter $\mathrm{Hg}$, die in die Grenzen normaler Blutdruckschwankungen fallen. Nur in 4 Fällen von alimentärer Leukopenie kam es zu einer Blutdrucksenkung von mehr als Io $\mathrm{mm} \mathrm{Hg}$ (bis $25 \mathrm{~mm} \mathrm{Hg}$ ). GröBter Tiefstand der Leukocytenzahlen und des Blutdruckes traten nicht immer gleichzeitig auf.

IV. Der refraktometrische Index sank in den meisten Fällen mit alimentärer Leukopenie in geringem $\mathrm{Maße}$ und zwar im Durchschnitt um $0,36 \%$, ein halb Prozent nur einmal stark uibersteigend $\left(\mathrm{I}, \mathrm{O}_{5} \%\right)$.

Wegen der dargelegten theoretischen Gründe und der infolge ihrer Undeutlichkeit und Unzuverlässigkeit praktisch unbrauchbaren Resultate ist die hämollastische Krise als Hilfsmittel für die klinische Diagnostik von Leberkrankheiten abzulehnen. (Aus der II. inn. Abtlg. des städt. Krankenhauses Moabit-Berlin [Geh. Rat Zinn].)

\section{KASUISTISCHE MITTEILUNG.}

\section{EIN FALL VON CEREBELLARER ENCEPHALITIS'1).}

$$
\text { Von }
$$

\section{Dr. Ladislaus Frigyér.}

Die cerebellare Lokalisation der Encephalitis erscheint ihrer Seltenheit halber der Veröffentlichung wert. In den wenigen von E. Redlich, Z Weigenthal, Hilgermann und Sarbó beobachteten Fällen trat die Erkrankung des Kleinhirns gleichzeitig mit der Lähmung der Hirnnerven auf, war aber nicht die Haupterscheinung des Krankheitsbildes. Da die Beobachtungen größtenteils aus der Zeit der akuten Encephalitisepidemie stammten, wissen wir nichts von den weiteren Schicksalen der Kranken. Wir sahen und erfahren es auch aus der Literatur, da $\beta$ bei einer nicht geringen Anzahl der Kranken der verlaufenen Epidemie ein Defekt zurückblieb, der auffallend an die Parkinsonsche Erkrankung erinnert. In dem $z \mathfrak{u}$ meiner Beobachtung gelangten Falle begann die Krankheit mit den typischen Symptomen der akuten Encephalitis (Schlafsucht, Sopor usw.), nach deren Ablauf ein Zustand hinterblieb, den ausgesprochene cerebellare Ataxie, allgemeine Muskelschwäche und Hypotonie kennzeichnete, im schroffen Gegensatze zu dem oben erwähnten Parkinsonartigen Symptomenkomplex.

In den beiden Fällen ist der Tonusapparat der Muskulatur erkrankt. Die Erfahrungen der letzten Encephalitisepidemie zeigten, daß bei der Innervation des Muskeltonus das Corp. striatum beteiligt ist. Angeblich entspringt diese Nervenbahn aus den subcorticalen Ganglien. Daß sich das Kleinhirn bei der Aufrechterhaltung des Muskeltonus beteiligt, ist eine altbekannte Tatsache. Diese Bahn entspringt"dem Nucleus dentatus und gerät durch das Brachium conjunctivum zum Nucleus ruber, von wo sie via Tract. rubrospinalis das Rückenmark erreicht. Tatsache ist, daß die Läsion der subcorticalen Ganglien die Rigidität der Muskulatur verursacht - ob dies auf Tonusstörung beruht, ist noch zweifelhaft, bei Erkrankung des Kleinhirns entwickelt sich im Gegensatze eine Hypotonie der Muskulatur. In meinem Falle scheinen alle Anzeichen auf die Erkrankung jenes Teiles des Tonusapparates zu deuten, der durch das Kleinhirn exhalten wird.

S. I., I 8 jähriges Mädchen. Vor acht Monaten erkältete sie sich angeblich und fieberte durch vier Wochen, sie litt an heftigen Kopfschmerzen. Bald darauf erkrankte sie an einer Lungenentzündung. In der ersten Woche der Erkrankung schlief sie Tag und Nacht; zur Einnahme der Mahlzeiten wurde sie von ihren Angehörigen geweckt. Nach Ablauf des Fiebers hatte sie Doppeltsehen, gestorte Sprache, Hände und Füße wurden so schwach, daß sie sie kaum gebrauchen konnte. Nach Wochen besserte sich der Zustand, das Doppeltsehen schwand vollkommen, die Extremitäten wurden kräftiger. Im Bette aufsitzend konnte sie das Gleichgewicht nicht behalten, der Rumpf bewegte sich hin und her. Gegenwärtig klagt sie hauptsächlich über Taumeln und starke Unsicherheit beim Gehen - kann sich ohne Hilfe nicht fortbewegen - und Ungeschicklichkeit in den Händen.

Der Nervenstatus der mittelmäßig entwickelten fieberfreien Kranken, deren innere Organe ohne besonderen Befund waren,

1) Vorgestelit im König1. Ung. Arrzteverein in Budapest am 22. Oktober I92I. ist folgender: Pupillen gleichmäßig, rund, reagieren gut. Rechts geringer Lagophthalmus, Kopfnerven sonst intakt. Kein Nystagmus, Augenhintergrundbefund normal. Patellar- und Achillesreflexe sind auch mit Griff nicht auslösbar. Die Sehnen und Periostreflexe der oberen Extremitäten sind schwach auslösbar, gleichmäßig. Bauchreflex vorhanden, keine päthologischen Reflexe.

Die oberen und unteren Extremitäten sind hochgradig hypotonisch. Die unteren Extremitäten können in liegender Stellung fast bis in die Höhe des Kopfes gehoben werden. Die motorische Innervation der Muskulatur zeigt keine Abweichung, doch sind sämtliche Muskeln des Körpers auffallend kraftlos, welchen Zustand wir als paretisch oder nach MANN eher als asthenisch bezeichnen können. Die elektrische Erregbarkeit normal, ebenso die oberflächliche und tiefe Empfindung. Der Gang der Kranken weist auf eine ausgesprochene Ataxie cerebellarer Natur. Durch die Gleichgewichtsstörung pendelt der Körper beim Gehen; in fortwährender Angst umzufallen, versucht die Kranke durch Armbewegung das Gleichgewicht zu erhalten. Wenn man ihr beim Gehen behilflich ist womit die Gleichgewichtsstörung gewissermaßen kompensiert wird - , bessert sich der Gang auffallend. In beiden Armen ausgesprochene Adiadochokinese. Ein analoges Symptom ist auch in der Sprache $z \mathfrak{u}$ beobachten, die langsam und schleppend ist (Adiadochokinesis lingualis, Bradylalia). Gewichtssinn normal, BrachRomberg + ; die Kranke fällt am häufigsten nach hinten. BárányVersuch gibt normale Reaktion. Kein spontanes Vorbeizeigen.

Die Kranke wurde nach Verlauf von drei Monaten neuerdings untersucht, der Befund zeigte keine Veränderungen.

Zusammenfassend ist der Nervenbefund folgender: Allgemeine Muskelasthenie und Hypotonie, Areflexie der unteren Extremitäten, cerebellare Ataxie, Adiadochokinesis und Adiadochokinesis lingualis, Symptome, die wir auch bei Kleinhirngeschwulsten beobachten, allerdings am häufigsten als halbseitige Erscheinung. Obzwar die Läsion der zentripetalen und zentrifugalen Bahnen (Tractus spinocerebellaris ventr. et dors., Tract. rubrospinalisvestibulospin.), die die Reflexbogen des Kleinhirns als Koordinationszentrum, darstellen gleichfalls solche Symptome verursachen, ist meiner Ansicht nach der Herd der Erkrankung in unserem Falle eher in der beiderseitigen Kleinhirnhemisphäre zu suchen.

Der Uxsprung der Krankheit könnte auch Sclerosis multiplex sein, doch spricht. - neben vielen anderen Symptomen - hauptsächlich der akut fieberhafte und für Encephalitis so charakteristische Beginn dagegen.

Ich halte obigen Fall der Veröffentlichung wert, da wir durch diesen eine neue Art der Encephalitisinvaliden kennen Iernten. Hier ist auch ein Tonusapparat beteiligt, aber mit Symptomen, die ganz im Gegensatze zu denen stehen, die wir bei den Parkinsonähnlichen Encephalitisinvaliden beobachten. Eigentümlicherweise greift das noch unbekannte Virus der Encephalitis mit großer Affinität den Tonusapparat an und ist die Läsion meistens doppelseitig. (Aus der II. internen Universitätsklinik in Budapest [Direktor: Dr. Ernst Jendrassik o. ö. Proffessor +].)

Literatur: OPPENHEIM: Hdb. d. Neurologie. - SCHILDER: Dtsch. Zeitschr. f. Nervenheilkunde, 64. - E. REDLICH: Neurologisches Zentribl. Igr8, S. 298. ZWEIGENTHAL: Med. Klinik I920, Nr. 44. - HIIGERMANN: Med. Klinik I920 Nr. $x$. - SARBO- Orvosi Hetilap $192 x$. 